\title{
EchoGéo
}

21 | 2012

Pays émergents

Séoul : la transition " sur le vif » des espaces publics de proximité (1995-2010)

\section{Valérie Gelézeau}

\section{OpenEdition}

1 Journals

Édition électronique

URL : https://journals.openedition.org/echogeo/13055

DOI : 10.4000/echogeo.13055

ISSN : 1963-1197

Éditeur

Pôle de recherche pour l'organisation et la diffusion de l'information géographique (CNRS UMR 8586)

Référence électronique

Valérie Gelézeau, «Séoul : la transition «sur le vif » des espaces publics de proximité (1995-2010) », EchoGéo [En ligne], 21 | 2012, mis en ligne le 10 octobre 2012, consulté le 10 août 2021. URL : http:// journals.openedition.org/echogeo/13055; DOI : https://doi.org/10.4000/echogeo.13055

Ce document a été généré automatiquement le 10 août 2021.

EchoGéo est mis à disposition selon les termes de la licence Creative Commons Attribution - Pas d'Utilisation Commerciale - Pas de Modification 4.0 International (CC BY-NC-ND) 


\title{
Séoul : la transition « sur le vif » des espaces publics de proximité (1995-2010)
}

\author{
Valérie Gelézeau
}

1 Comment la transformation des formes du logement change-t-elle les pratiques quotidiennes? La mutation des espaces domestiques (privés) a-t-elle un effet sur l'usage des espaces publics?

2 Saisie ici dans 8 illustrations, l'évolution "sur le vif», en une quinzaine d'années (1995-2010), d'un quartier du péricentre de Séoul (Singongdeok-tong, dans l'arrondissement de Map'o) illustre quelques uns des changements qui peuvent affecter les espaces publics de proximité - ceux de la quotidienneté, à l'échelle du quartier ou de l'îlot résidentiel.

3 Les photographies 1 à 4 ont été prises en 1996. La photographie 1 présente un périmètre désigné de rénovation urbaine en contrebas d'une colline. L'îlot à rénover, au premier plan, comprend un groupe de maisons datant des années 1950, dont la forme (toits de tuile recourbés, lourdes portes en bois, minuscules ouverture des fenêtres) et la structure (plan en $\mathrm{L}$ ou en $\mathrm{U}$ avec une cour intérieure) reprend certaines normes architecturales du début du XXe siècle. Ces maisons, sur un seul niveau, sont connectées à un labyrinthe de ruelles piétonnes. Le cœur de l'îlot est exclusivement résidentiel et les services et commerces (voir le pressing au premier plan) sont rejetés en périphérie de l'îlot, sur les artères principales, assurant la transition avec les îlots voisins. Dans cet archétype résidentiel, la fonction d'espace public de proximité est assurée par les ruelles qui constituent aussi une forme d'extension des espaces privés pour la communauté de voisinage. 
Illustration 1 - Le périmètre de rénovation de Singondeok-tong en 1996

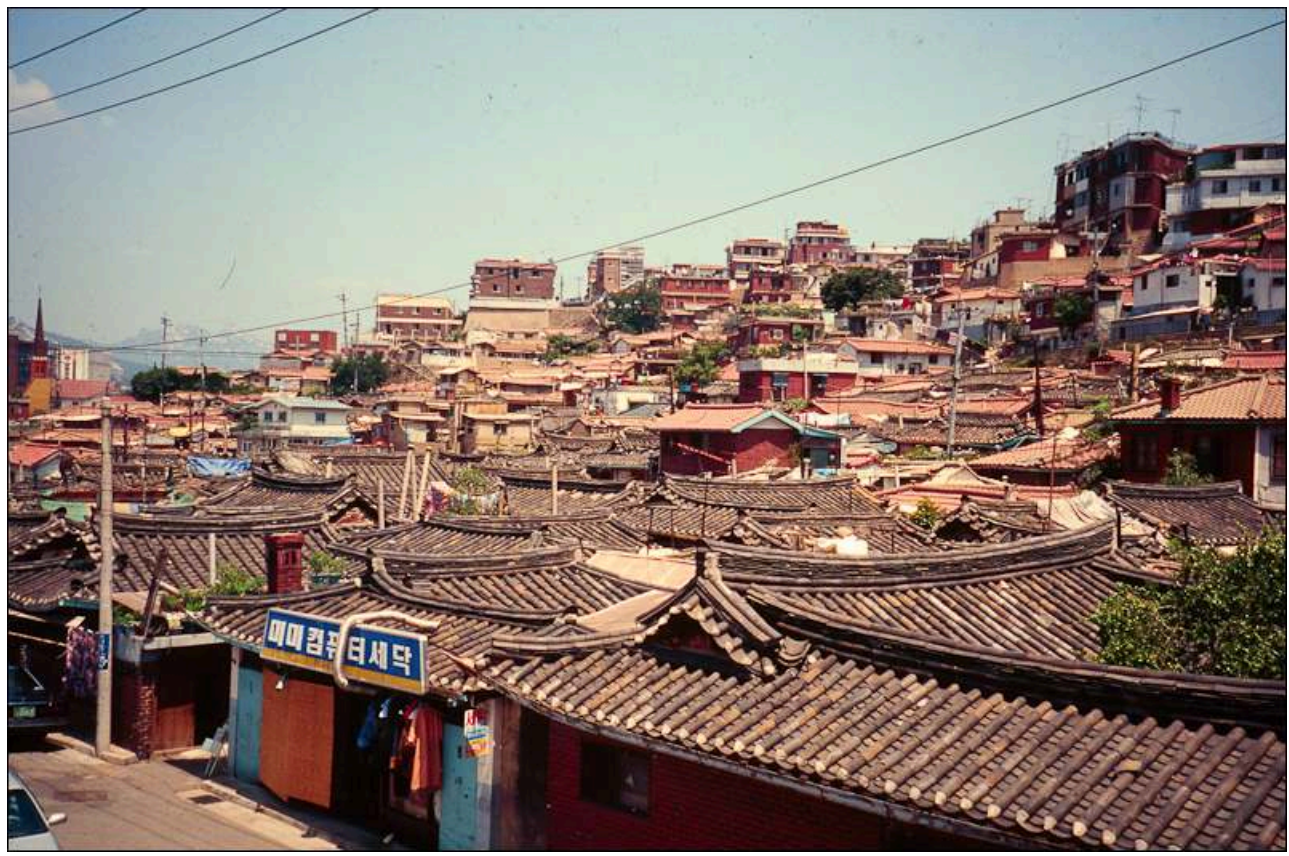

Auteur : Valérie Gelézeau, 1996.

4 Les photographies 2, 3 et 4, toutes prises dans ce quartier entre 1996 et 1999, illustrent cet usage d'entre-deux de la ruelle : espace semi-privé qui est aussi un espace public de proximité, où les femmes se retrouvent pour éplucher les courgettes en échangeant les nouvelles (illustration 2), où les enfants circulent en tricycle (ce qui signifie qu'il n'y a pas de danger de circulation, illustration 3), voire où l'on laisse sans surveillance sécher le piment à l'automne (illustration 4). 
Illustration 2 - L'usage semi-privé d'une impasse dans le « labyrinthe » des ruelles à Shingondeoktong

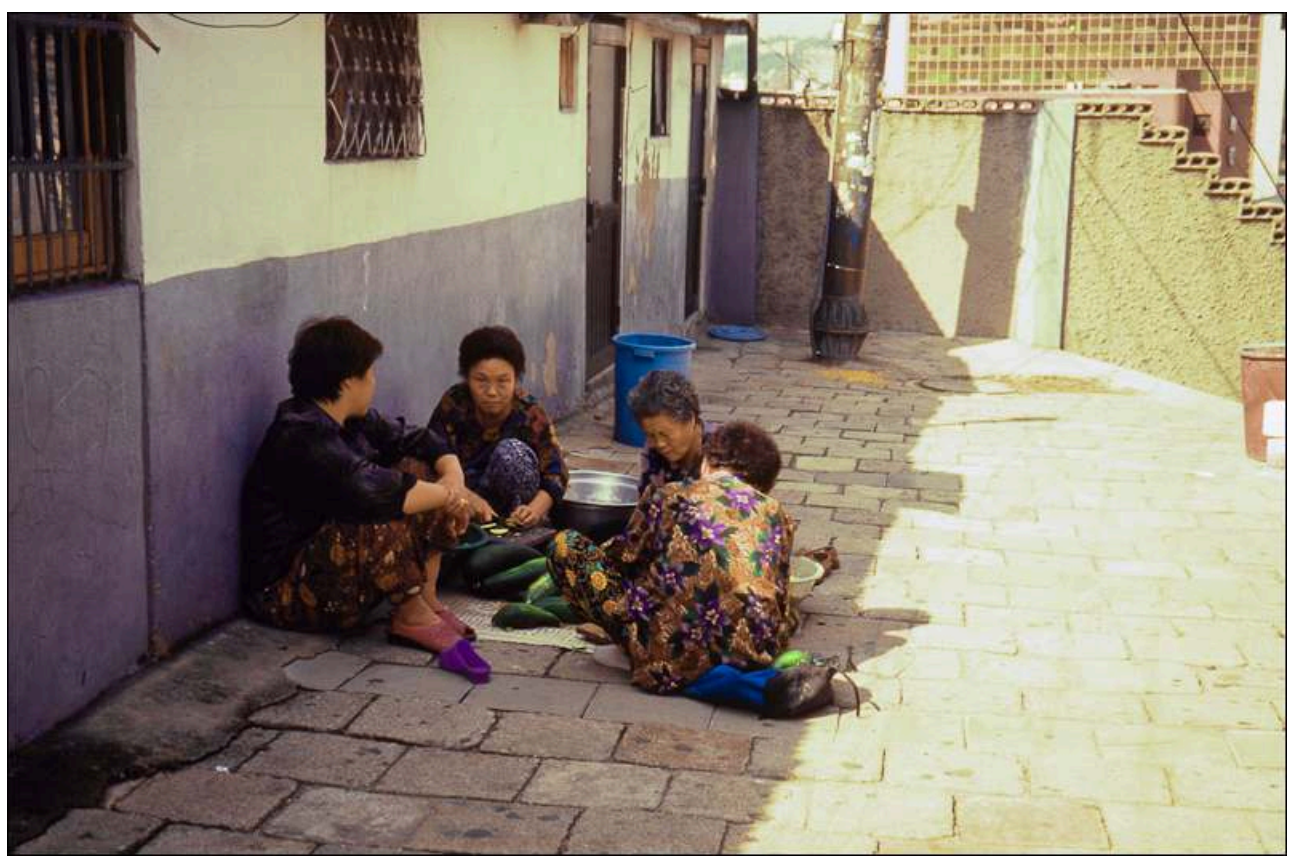

Auteur : Valérie Gelézeau, 1996.

Illustration 3 - À Shingongdeok-tong en 1996, la ruelle est un terrain de jeu pour enfants

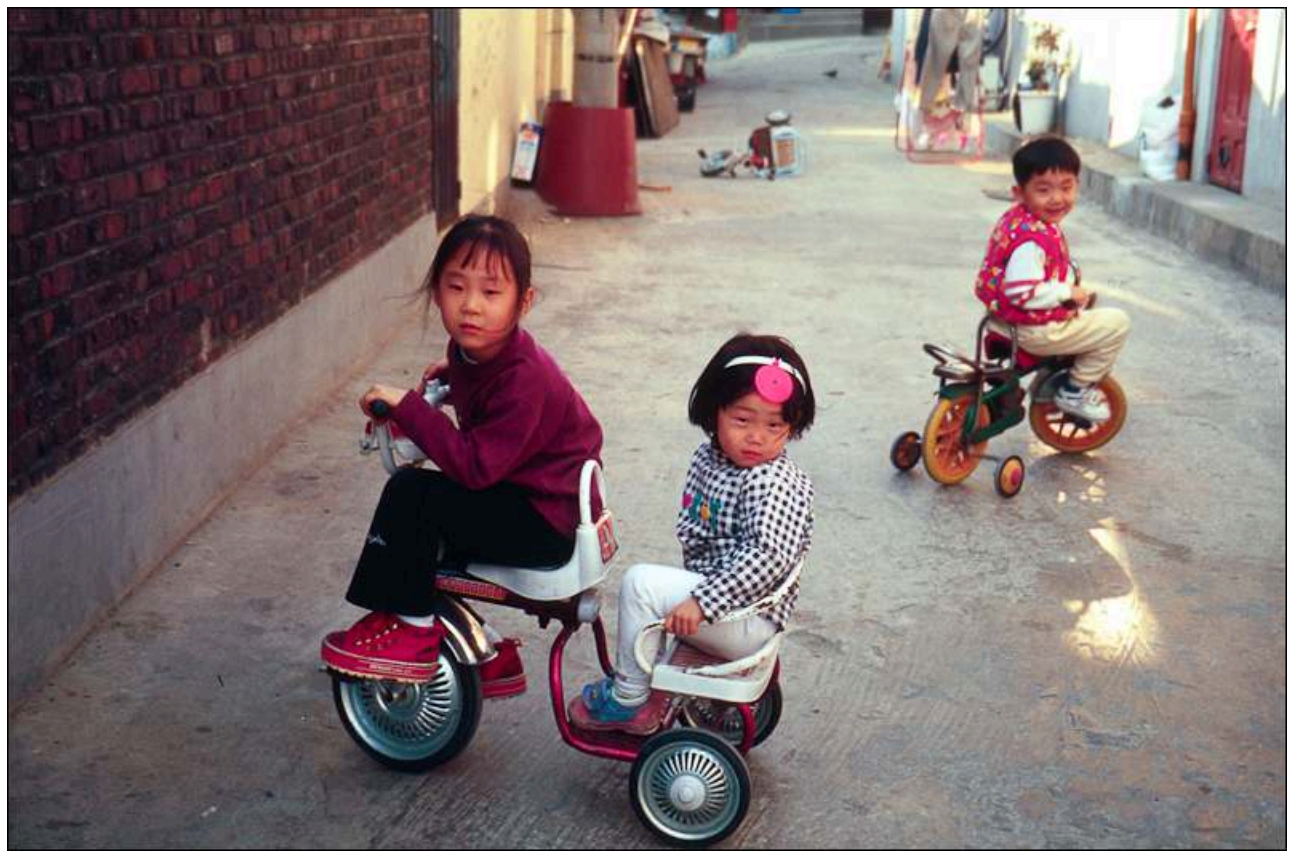

Auteur : Valérie Gelézeau, 1996. 
Illustration 4 - À Shongongdeok-tong, des piments sèchent dans une ruelle qui apparaît comme une extension de l'espace privé

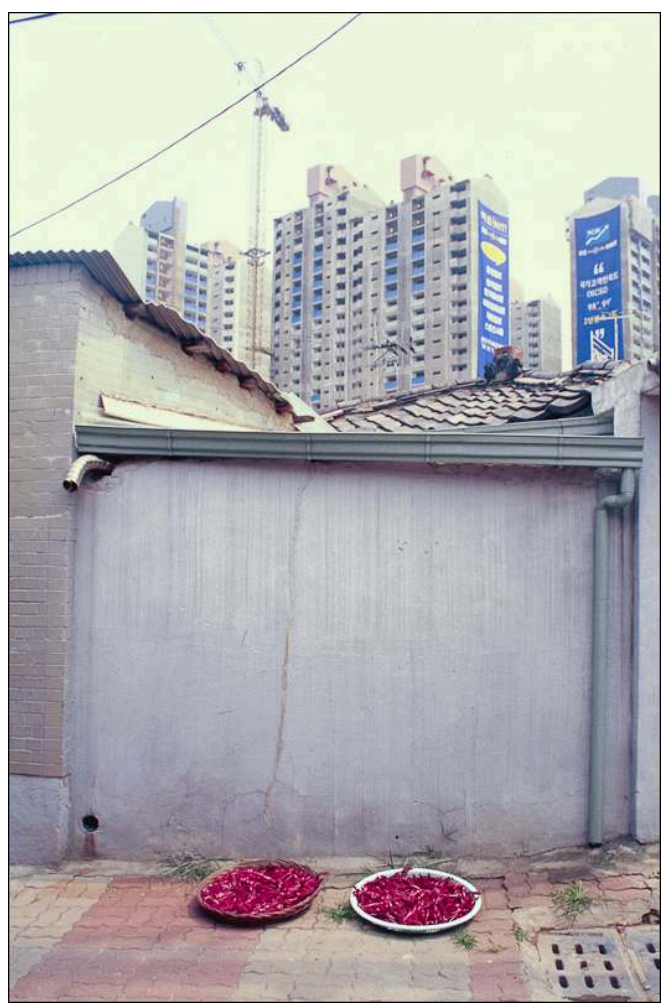

Auteur : Valétie Gelézeau, 1999.

5 En 2000, cet îlot avait été reconstruit sous la forme d'un complexe résidentiel comprenant une dizaine d'immeubles de 25 à 28 étages (illustration 5). 
Illustration 5 - Le périmètre de rénovation de Singondeok-tong en 2000

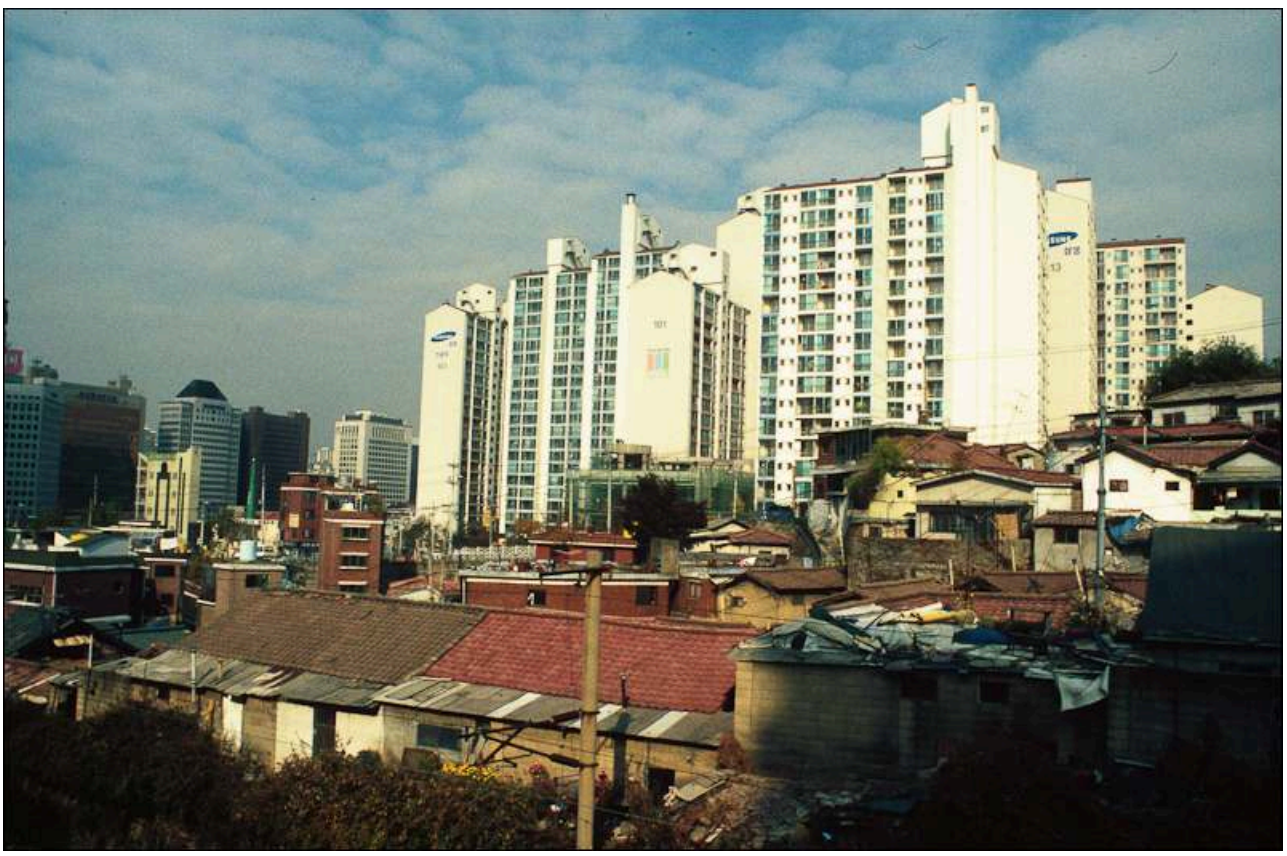

Le périmètre de rénovation de l'illustration 1 a été reconstruit par une filiale construction du conglomérat Samsung.

Auteur : Valérie Gelézeau, 2000.

6 La photographie 6, prise en 2010, montre de nouvelles transformations, encore plus profondes : les immeubles aux toits rouges de ce premier îlot rénové qui dominait de sa masse d'autres quartiers dégradés voués à la rénovation en 2000, apparaissent désormais comme «nanifiés " par les tours collectives de grande hauteur construites à la fin de la décennie. 


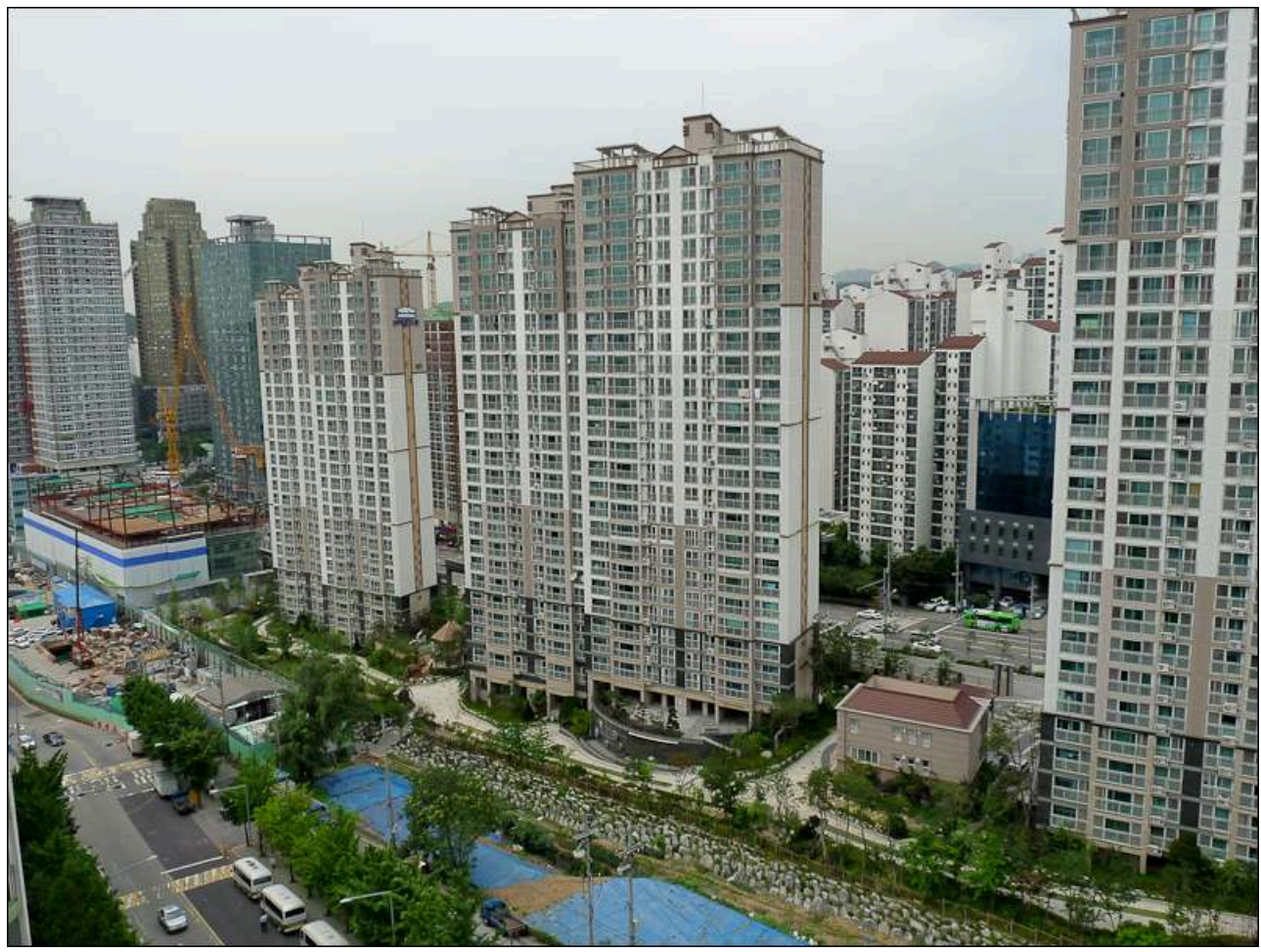

L'îlot dégradé au premier plan de l'illustration 5 a été reconstruit (tours de grande hauteur au premier plan).

Auteur : Valérie Gelézeau, 2010.

7 Bien que des différences importantes en termes de filière de production, de conception, de contenu social et d'image distinguent le premier complexe résidentiel de ces tours collectives plus récentes et plus luxueuses, les espaces publics de proximité relèvent de la même logique de conception d'équipements adaptés aux populations résidentes : soit des espaces ouverts créés pour cette fonction, comme les terrains de jeu pour enfants (illustration 7), parfois agrémentés de jeux d'eau pour les plus récents (illustration 8); soit des espaces communs fermés comme la maison des personnes âgées. A cela s'ajoutent les clubs divers fréquentés par les femmes résidentes des grands ensembles (club de sport, de danse traditionnelle, de musique, etc.). Dans ces nouveaux complexes résidentiels, on observe parfois le maintien de pratiques traditionnelles, par exemple l'usage des nattes et des petites estrades posées en lisière d'un terrain de jeu où s'installent les grands-mères pour surveiller leurs petits-enfants. Mais ces pratiques liées aux postures corporelles de la «vie près du sol » dans des espaces domestiques traditionnellement exempts de meubles hauts tendent à disparaître avec les personnes qui en sont les dépositaires. 
Illustration 7 - À Sadang-dong, une scène classique dans un grand ensemble : le terrain de jeux pour enfants

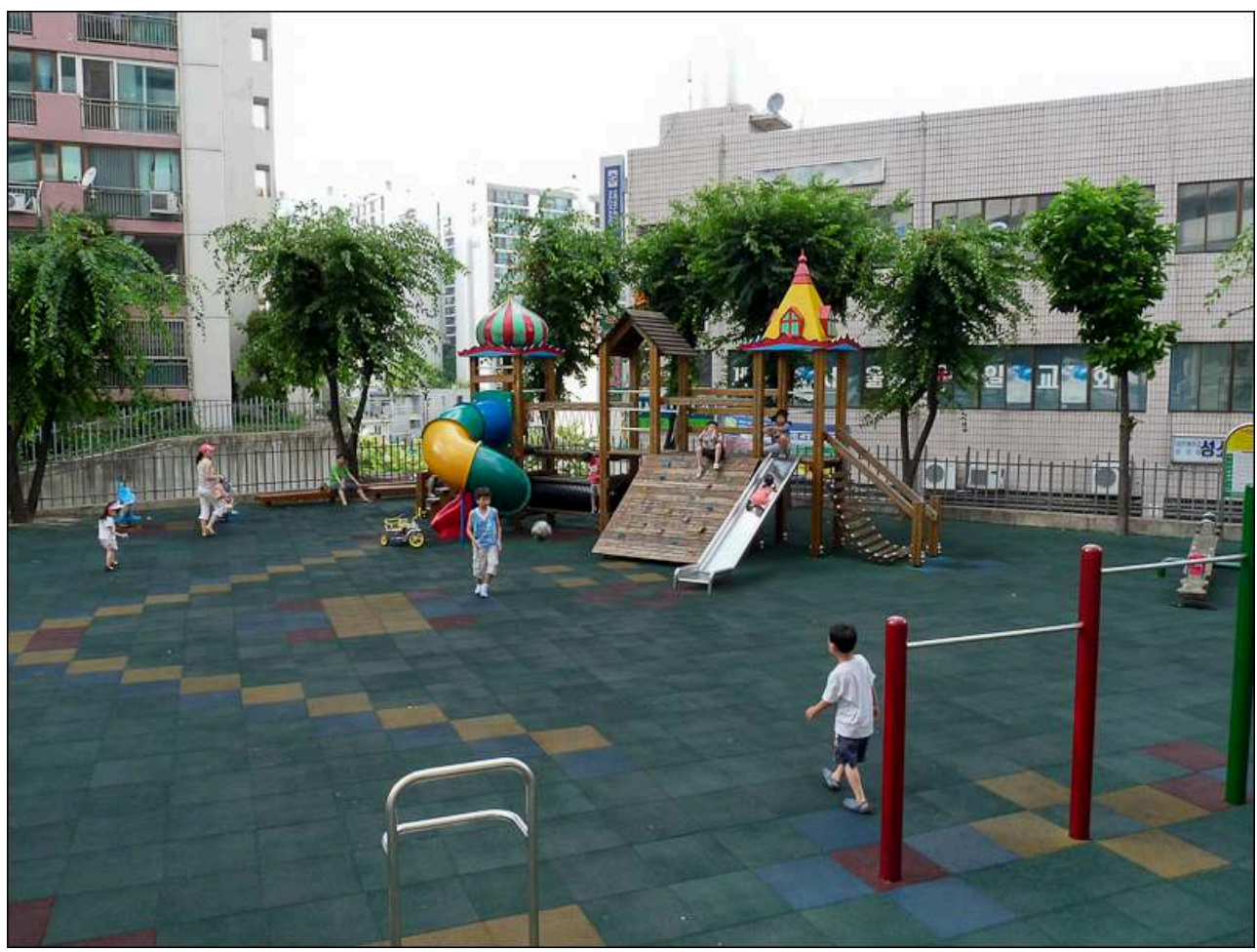

Auteur : Valérie Gelézeau, 2010

Illustration 8 - À Palsan-dong, l'aménagement récent des grands ensembles avec des fontaines et des jeux d'eau très appréciés des enfants en été

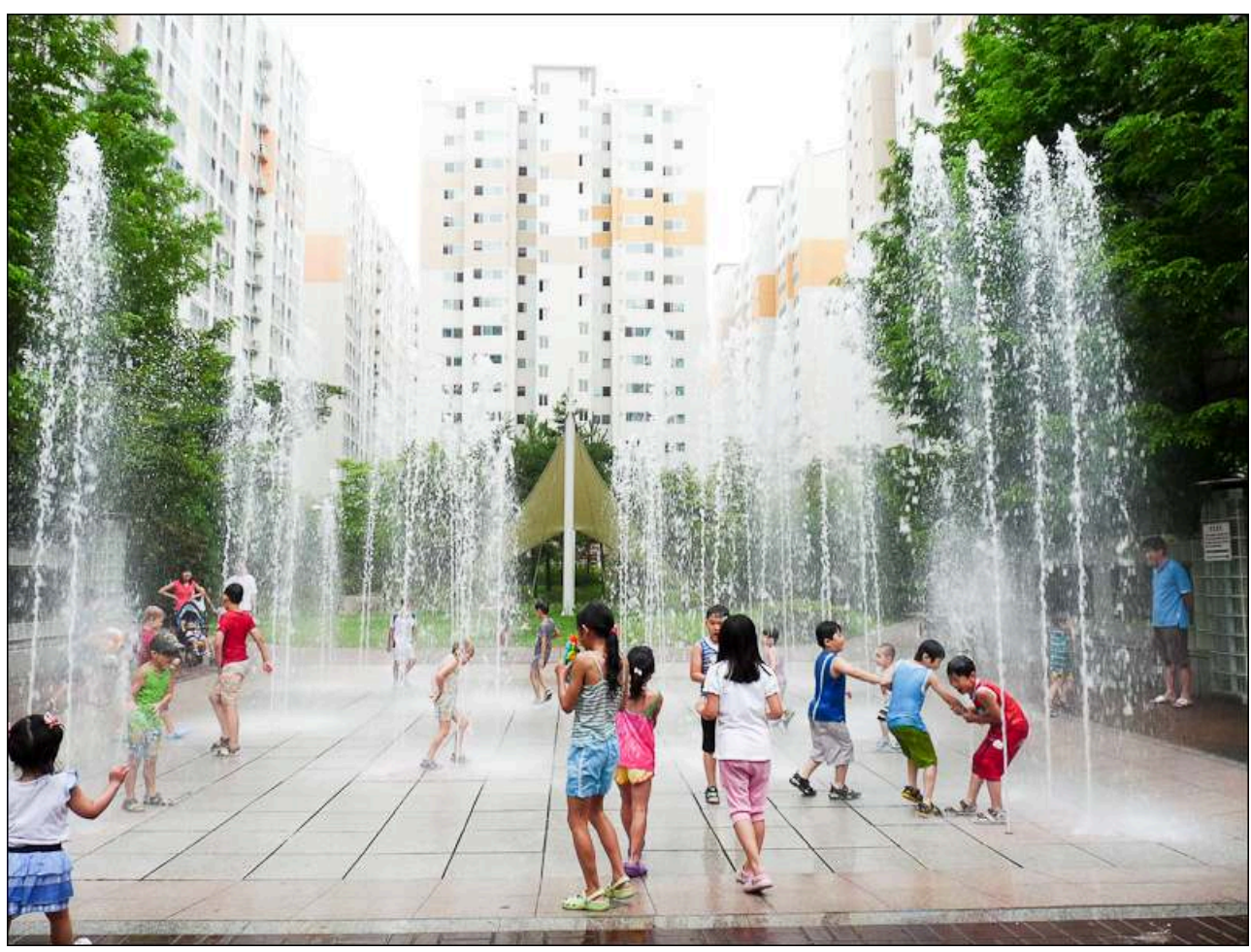

Auteur : Valérie Gelézeau, 2010 


\section{AUTEUR}

\section{VALÉRIE GELÉZEAU}

Valérie Gelézeau, valerie.gelezeau@gmail.com, est Maîtresse de conférences à l'EHESS, membre de l'UMR 8173 Chine, Corée, Japon (CNRS-EHESS-Paris 7). Elle a publié récemment

- Gelézeau V., 2010. Espoirs et désillusions de la décennie du « rayon de soleil ». Critique internationale, octobre-décembre 2010, Thema « Coopérations coréennes, 1998-2008 », p. 9-20.

- Gelézeau V., 2011. The inter-Korean border region - 'meta-border' of the Cold War and metamorphic frontier of the peninsula. In Doris Wastl-Walter (ed), The Ashgate Research Companion to Border Studies. Oxon (UK), Ashgate, 2011, p. 325-348.

- Gelézeau V., 2011. Séoul. Autrement, collection « Mégapoles », Paris, 88 p. 